\title{
Trends for conception rate of Holsteins over time in the southeastern United States
}

\author{
C. Huang, ${ }^{* 1}$ S. Tsuruta, ${ }^{*}$ J. K. Bertrand, ${ }^{*}$ I. Misztal, ${ }^{*}$ T. J. Lawlor, $†$ and J. S. Claył \\ *Department of Animal and Dairy Science, University of Georgia, Athens 30602 \\ †Holstein Association USA Inc., Brattleboro, VT 05301 \\ ‡Dairy Records Management Systems, Raleigh, NC 27603
}

\section{ABSTRACT}

The purpose of this study was to estimate trends in conception rate (CR) of Holsteins in the southeastern United States over time across month by milk production level and month by days in milk (DIM) subclasses. Data were obtained from Dairy Records Management Systems (Raleigh, NC) and included service records from 10 states (Virginia, Kentucky, North Carolina, South Carolina, Tennessee, Georgia, Florida, Alabama, Mississippi, and Louisiana). After eliminating records with lactation $>1$ and uncertain and extreme records (records without calving or birth date, with days to service after calving $<21$ or $>250$, or without next calving date), the final data set included 827,802 artificial insemination service records for 424,513 cows born from 1985 to 2000, and in 2,953 herds. Effects included in the model were year of birth (1985 to 1989, 1990 to 1994, 1995 to 2000), DIM class, milk production level (high, medium, low based on SD), service month, the covariate of cow age at calving, and 2- and 3-way interactions. Over time, an increase was observed for milk production and an overall decline in CR occurred. Examination of month by milk production subclass least squares means showed that in cool months (November to April) the deterioration of CR over time was small for low and medium milk production cows and virtually none for high-producing cows. However, in other months (May to June), there was a large decline over time in CR for cows in all milk production level subclasses. The trends in CR by DIM subclasses were examined for the months of February, May, June, and August. There was a general increase in CR with increasing DIM for all months within all birth-year groups. The months of February and August were somewhat similar for CR up to 175 DIM for the different birth-year groups. Much larger differences over time were observed for the months of May and June, and it appeared that for these 2 mo,

Received December 17, 2008.

Accepted May 7, 2009.

${ }^{1}$ Corresponding author: pang $127 @$ uga.edu cows in recent periods did not return to the same level of performance as cows in earlier periods. It may be that there has been a decline over time in the ability of cows to handle the onset of heat stress or the switch to pasture-based management systems.

Key words: conception rate, fertility, trend, heat stress

\section{INTRODUCTION}

A decline in fertility over time in US Holsteins has been reported by several studies (Lucy, 2001; Washburn et al., 2002; de Vries and Risco, 2005). A decrease in cow fertility has been associated with increased milk production (Dematawewa and Berger, 1998; Lucy, 2001; Pryce and Harris, 2004). Lucy (2001) reported that other factors, including season of calving, were equally important contributors to fertility decline. López-Gatius (2003) found that milk yield increased over time and that season had an effect on yearly trends in reproduction, with much steeper declines in fertility in the hot season compared with the cool season. de Vries and Risco (2005) reported that pregnancy rate after a 70-d voluntary waiting period (VWP) was greater in the winter compared with the summer season, and that the absolute decline over time for this measure of reproduction was similar over both seasons. However, in the same study, it was found that the ratio of pregnancy rate in the winter compared with the summer season has decreased over time, indicating that the relative effect of heat stress on fertility has increased over time.

Only selected parts of the United States experience long periods of heat stress. However, because of intense heat during the summer time and increased sensitivity to heat stress, the effect of heat stress on fertility has been observed as far north as Alberta, Canada (Brouk et al., 2007). Even a short period of heat stress may have a long-term effect on fertility (Morton et al., 2007).

The purpose of this study was to analyze individual inseminations using Dairy Herd Improvement records collected from 1985 to 2000 on Holsteins in 10 southeastern states to describe trends in conception rate 
(CR) over time across month by milk yield subclasses and across month by DIM subclasses.

\section{MATERIALS AND METHODS}

\section{Data}

Animal Care and Use Committee approval was not obtained for this study because field data were used. Insemination and production records from 1988 to 2004 were obtained from Dairy Record Management Systems (Raleigh, NC) and contained data from Virginia, Kentucky, North Carolina, South Carolina, Tennessee, Georgia, Florida, Alabama, Mississippi, and Louisiana. These states are hot and humid during a substantial part of the year. See Bohmanova et al. (2007) for monthly temperature-humidity averages in Georgia and Bohmanova et al. (2008) for heat-stress degree per year per state. Humid climates with lower temperatures such as in Georgia may create greater heat stress than climates with greater temperatures and low humidity such as Arizona because the cooling devices are less efficient at high humidity. The data editing procedures were similar to those used by Huang et al. (2008). Artificial insemination records without a birth date, calving date, service date, cow identification, and lactation number were removed. Multiple breedings only from primiparous cows were considered. Further edits eliminated service records with $<21$ or $>250$ DIM and first calving age $<20$ or $>36$ mo. To confirm a successful second-parity conception, a cow was required to have a subsequent calving record linked to a possible breeding event during her first lactation. Cows without this information were removed. For calving records that were linked to a first-lactation breeding event, a predicted last-service date was calculated from the calving date initiating the second lactation minus $280 \mathrm{~d}$. If the difference between the predicted service date and the observed last-service date was within $\pm 10 \mathrm{~d}$, the observed service date provided with the record was used as the successful conception date. If the difference between the predicted service date and the observed last-service date was between 10 and $70 \mathrm{~d}$ or between -10 and -70 $\mathrm{d}$, the predicted service date was used as the successful conception date. If the difference between the predicted service date and observed last-service date was greater than $70 \mathrm{~d}$ or less than $-70 \mathrm{~d}$, the record was removed. For each service recorded, the cow received a score of 1 if she conceived as a result of the service or a score of 0 if she failed to conceive. The resulting trait, called the $\mathrm{CR}$, provides the probability of conception for a particular service.

Three milk production levels (low, medium, high) were classified based on the average of the first 3 test-

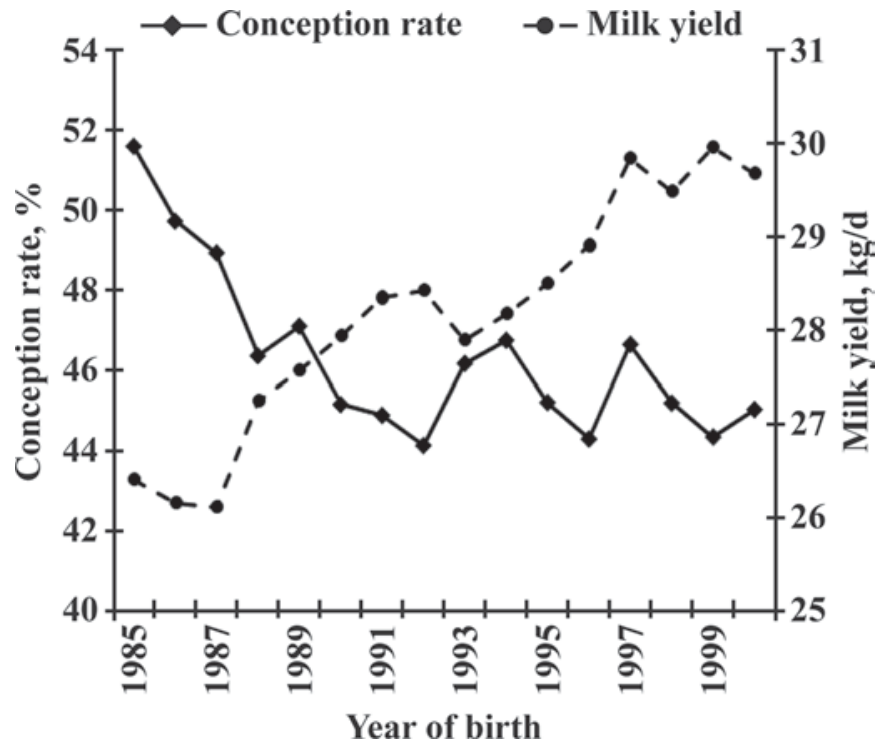

Figure 1. Mean conception rate and daily milk yield of first 3 test-day milking records from primiparous lactating cows over years of birth.

days during the first lactation because information was available only from the first 3 test-days. Cows were assigned to milk production levels based on the mean and $\mathrm{SD}$ within each year of birth $(<$ mean $-1 \mathrm{SD}$, between $\pm 1 \mathrm{SD}$ from mean, >mean $+1 \mathrm{SD}$ ) because test-day milk production increased over time (Figure 1). Cows without the first 3 test-day milk records were removed. After removing those years with small number of CR records, only cows born from 1985 to 2000 were used. The final data set consisted of 827,802 service records for 424,513 cows from 2,953 herds. The numbers of service records are shown in Table 1 by birth year, DIM group, service month, and milk production level. Table 2 shows the number of services per state. Table 3 presents the average milk in each production level per birth year.

\section{Statistical Analyses}

Data were initially analyzed using both the GLM procedure and the LOGISTIC procedure in SAS (SAS Institute, 1996). Included in the model were the effects of age at calving as a covariate, DIM interval group (from 25 to 250 d every $25 \mathrm{~d}$ ), service month, milk production level (<mean $-1 \mathrm{SD}$, between $\pm 1 \mathrm{SD}$ from mean, >mean + 1 SD), year of birth group (1985 to 1989, 1990 to 1994, 1995 to 2000), and all possible 2 -way and 3-way interactions. Because the LOGISTIC procedure was time and memory intensive and the initial results from the LOGISTIC procedure produced similar trends to those from the GLM procedure, the 
Table 1. Number of service records in year of birth, days in milk group, service month, and milk production level

\begin{tabular}{lr}
\hline Item & Records, $\mathrm{n}$ \\
\hline Year of birth & \\
1985 & 9,923 \\
1986 & 17,078 \\
1987 & 18,479 \\
1988 & 20,460 \\
1989 & 21,146 \\
1990 & 23,981 \\
1991 & 23,243 \\
1992 & 29,190 \\
1993 & 40,089 \\
1994 & 38,707 \\
1995 & 34,194 \\
1996 & 32,470 \\
1997 & 30,273 \\
1998 & 31,871 \\
1999 & 30,958 \\
2000 & 22,451 \\
Milk production level & \\
Low & 120,958 \\
Medium & 573,122 \\
High & 133,722 \\
DIM group & \\
$21-50$ & 45,620 \\
$51-75$ & 189,712 \\
$76-100$ & 176,182 \\
$101-125$ & 131,969 \\
$126-150$ & 96,856 \\
$151-175$ & 70,349 \\
$176-200$ & 50,994 \\
$201-225$ & 37,058 \\
$226-250$ & 29,062 \\
Service month & \\
January & 87,154 \\
February & 70,717 \\
April & 68,712 \\
May & 58,856 \\
June & 51,904 \\
July & 43,450 \\
August & 47,208 \\
Octoberber & 56,482 \\
November & 69,174 \\
December & 87,248 \\
\hline & 94,705 \\
& 92,192 \\
\hline
\end{tabular}

GLM procedure was used to conduct all subsequent analyses, and the resulting least squares (LS) means from this procedure are presented.

\section{RESULTS AND DISCUSSION}

All CR calculated in this study were most likely overestimated because cows with incomplete information or that failed to conceive were eliminated; more accurate editing was problematic using the information that was available. All main effects in the model were highly significant $(P<0.001)$. The only 2 -way interaction that was not significant $(P<0.01)$ was the interaction between DIM interval group with year of birth group; however, this interaction was nearly significant $(P<$
$0.06)$. The only significant 3 -way interaction $(P<0.01)$ was between year of birth, service month, and milk production level. Subsequently, nonsignificant effects were removed from the analyses. Phenotypic trends from 1985 to 2000 show that milk yield/d increased, whereas CR decreased from 1985 to 1992 and somewhat leveled off from 1993 to 2000 to rates below those observed in 1985 to 1987 (Figure 1). The general decline in CR for southeastern states agrees with several studies that reported an increase in milk production with a corresponding decline in several different fertility traits (Silvia, 1998; Washburn et al., 2002; de Vries and Risco, 2005).

Figure 2 provides a graph of the CR LS means for service month by birth-year group subclasses. In general, CR was highest and nearly constant across the months of December to April. Conception rate then began to decline in May until it reached its lowest point in August; it then began recovering but did not reach January to April levels until December. An examination of Figure 2 also reveals that small to no decrease in CR over time was observed from August to April, whereas CR decreased noticeably over time in May and June.

Figure 3 provides graphs of the LS means for CR for service month by birth-year group subclasses for high, medium, and low milk production levels in the southeastern United States. This information allows one to observe if deterioration in CR over time was the same for high- versus low-producing cows. In general, over all birth-year groups and service months, CR decreased as milk production increased. A slight decrease in $\mathrm{CR}$ was observed over time for low-producing cows during the months of January to April. A smaller decrease in CR over time was seen for medium-producing cows, and no trend over time was apparent for high-producing cows during the same 4-mo span. However, during the months of May and June, there was a large decline in $\mathrm{CR}$ over time within all milk production groups. The absolute amount of decline over time during these 2 mo did not appear to be associated with increased milk production. For example, the decrease from the 1985

Table 2. Number of services per state

\begin{tabular}{lc}
\hline State & Number of services $(\%)$ \\
\hline Virginia & $100,356(30.67)$ \\
North Carolina & $38,180(11.67)$ \\
South Carolina & $15,691(4.80)$ \\
Georgia & $37,318(11.41)$ \\
Florida & $55,252(16.89)$ \\
Kentucky & $18,202(5.56)$ \\
Tennessee & $30,154(9.22)$ \\
Alabama & $9,924(3.03)$ \\
Mississippi & $13,377(4.09)$ \\
Louisiana & $8,714(2.66)$ \\
\hline
\end{tabular}


to 1989 birth-year group to the 1995 to 2000 birth-year group for low milk production cows during the month of May was from 61.5 to $51.4 \%$ (10.1 percentage units), whereas the decrease for high milk production cows was from 52.3 to $47.9 \%$ (4.4 percentage units). For the month of June, the absolute CR decline from the 1985 to 1989 birth-year group to the 1995 to 2000 birth-year group for the low and high milk production levels were 12.9 and 8.7 percentage units, respectively.

The decline in CR over time was smaller but still evident into July and August, but during the month of September, the decline in CR over time was no longer apparent. The CR trends over time during October to December were similar to those during January to April, where a slight decrease in CR over time was observed for low-producing cows, but no time trend in CR appeared to exist for high-producing cows. López-Gatius (2003) found that milk production increased over a 10yr period in Holstein cows in Spain from 1991 to 2000. He also reported a very slight decrease in fertility over time during the cool season, whereas there was a dramatic drop in fertility over the 10-yr period during the warm season. Because cows in the current study were assigned to milk production levels within each year of birth, an increase in average milk production over time within milk production class was observed. The average 305-d milk yield increase for cows classified in the low, medium, and high milk production levels was 407, 818 , and $1,059 \mathrm{~kg}$, respectively, from the first birthyear group (1985 to 1989) to the last birth-year group (1995 to 2000). The high milk production class had the largest trend in milk production over time of the 3 milk production classes, yet it appeared to have the lowest decline in CR over time during the coolest months of

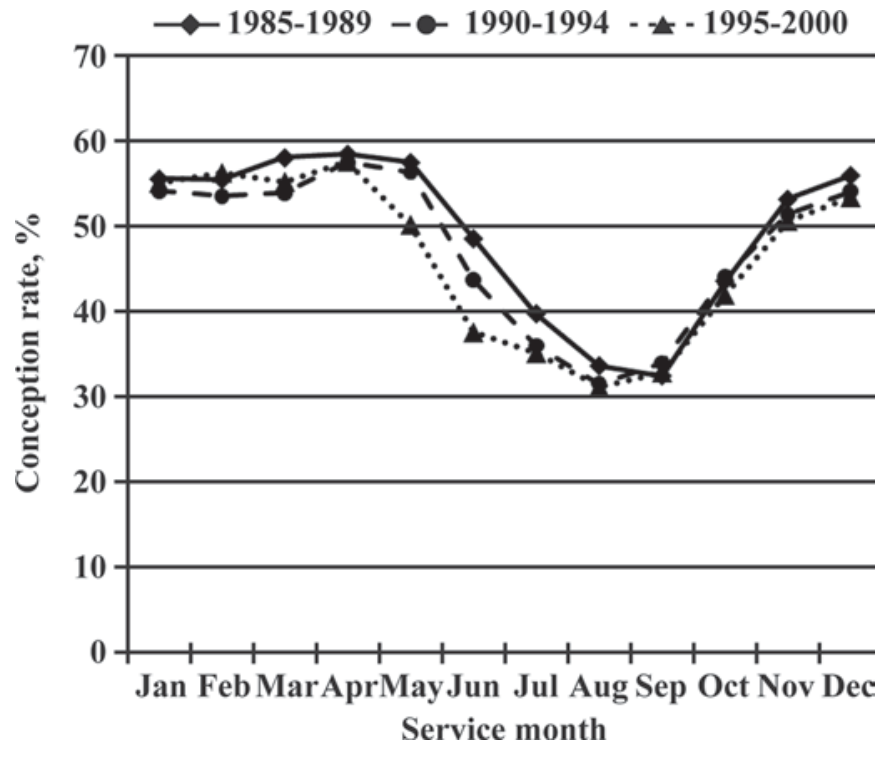

Figure 2. Least squares means of conception rates for service month by birth year subclasses for lactating Holstein cows in the southeastern United States.

the year (October to April). Huang et al. (2008) examined CR in dairy cows during 2000 to 2003 in Georgia and found that cows experienced a large decline in CR beginning in May and June. The levels of CR would then level off during July to September and increase again in October. The authors theorized that this drop could be caused by heat stress and management factors such as a switch to more pasture-based systems.

The drastic decrease in CR over time within all milk production groups during May and June observed in Figure 3 implies that negative consequences on fertility

Table 3. Mean daily milk production (kg) by birth year of cows classified as low, medium, and high producing (number of records in parentheses)

\begin{tabular}{lccc}
\hline & \multicolumn{3}{c}{ Milk production level $^{1}$} \\
\cline { 2 - 4 } Year of birth & Low & Medium & High \\
\hline 1985 & $19.00(2,650)$ & $26.40(12,030)$ & $34.41(2,844)$ \\
1986 & $18.71(4,572)$ & $26.10(21,569)$ & $34.00(5,235)$ \\
1987 & $18.64(5,081)$ & $26.07(23,339)$ & $33.96(5,862)$ \\
1988 & $19.58(5,640)$ & $27.20(27,145)$ & $35.34(6,723)$ \\
1989 & $19.49(6,021)$ & $27.49(27,766)$ & $36.15(6,704)$ \\
1990 & $19.70(7,010)$ & $27.96(32,631)$ & $36.40(7,833)$ \\
1991 & $20.19(6,929)$ & $28.35(32,510)$ & $36.81(7,473)$ \\
1992 & $20.12(8,871)$ & $28.51(40,288)$ & $36.83(9,616)$ \\
1993 & $19.58(11,451)$ & $27.93(53,499)$ & $36.45(12,662)$ \\
1994 & $19.58(10,724)$ & $28.22(51,417)$ & $37.14(12,044)$ \\
1996 & $19.77(9,933)$ & $28.52(46,660)$ & $37.52(10,511)$ \\
1997 & $20.12(9,572)$ & $28.95(45,574)$ & $37.77(10,263)$ \\
1998 & $21.05(8,339)$ & $29.92(40,635)$ & $38.88(9,335)$ \\
1999 & $20.46(8,872)$ & $29.53(43,995)$ & $38.43(9,926)$ \\
2000 & $20.93(8,849)$ & $30.05(43,189)$ & $38.99(9,497)$ \\
\hline
\end{tabular}

${ }^{1}$ Based on the first 3 test-day records. 


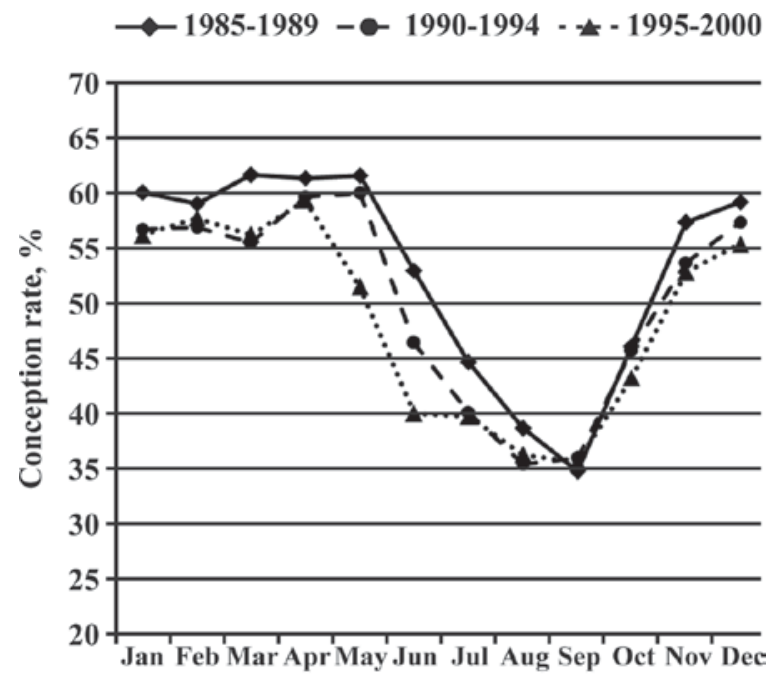

Service month; in low milk yield group

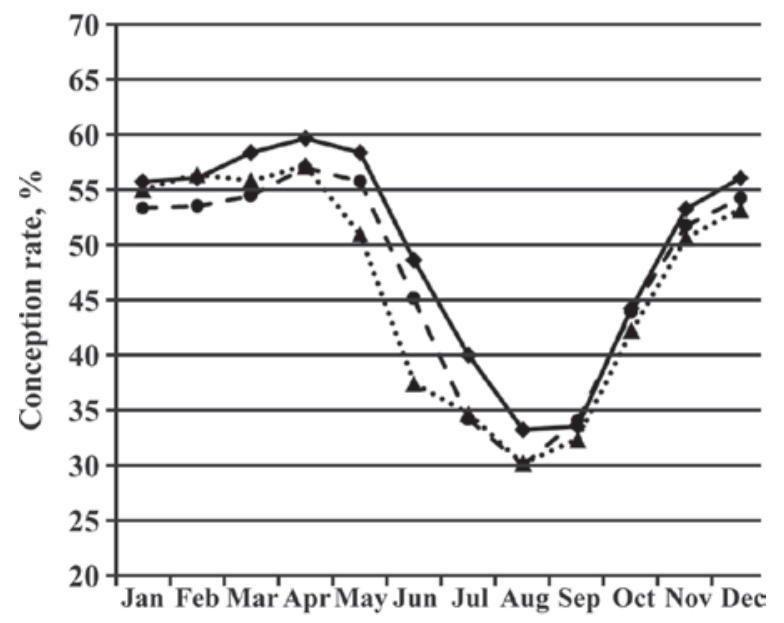

Service month; in medium milk yield group

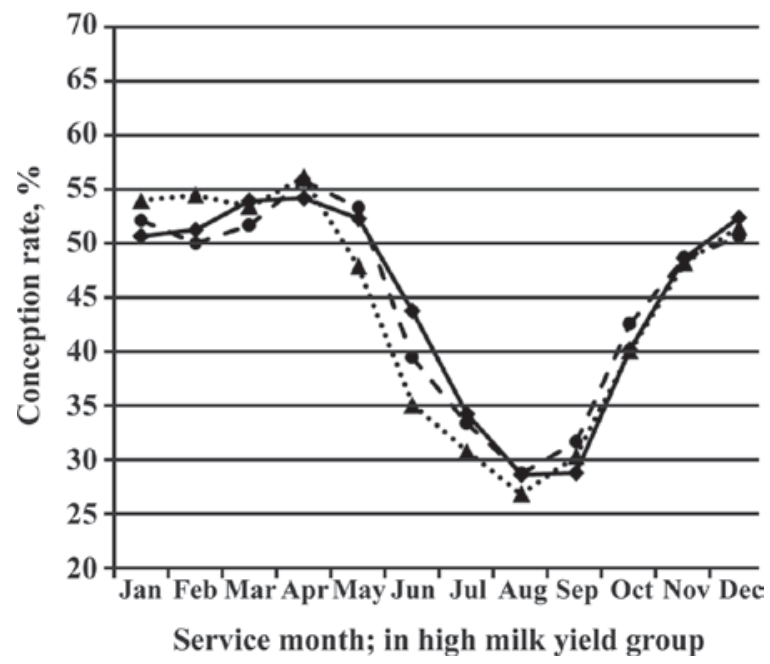

Figure 3. Conception rates for service month by birth-year group subclasses for high, medium, and low milk production groups in the southeastern United States. from increased energy demands due to increases in milk production may not be the sole explanation of why cows in the southeastern United States have declined in fertility over the years. Lucy (2001) reported in a review article that, even within comparable levels of milk yield, indicators of fertility in the United States declined from 1992 to 1999. He went on to state that "factors in addition to milk production are decreasing reproductive efficiency in US dairy cows." de Vries and Risco (2005) reported that the ratio comparing the summer season to the winter season for pregnancy rate decreased from $56 \%$ in the 1970 s to $35 \%$ in 2002 in Florida and Georgia, indicating that there has been an increase in the negative effect of heat stress over time.

One managerial response to the apparent decrease in fertility for modern Holstein cows has been to increase the VWP for breeding to beyond $70 \mathrm{~d}$ postpartum (de Vries and Risco, 2005). Huang et al. (2008) found that the increase in CR with increasing DIM in Georgia was seasonal in nature and that May was a month that showed a substantial improvement in CR from 25 to 50 to 75 to 100 DIM, whereas the months of February and August showed little improvement of CR with increasing DIM. However, the improvement was region dependent, and farmers cannot strongly increase VWP without compromising the calving interval.

Figure 4 provides CR for service month by DIM subclasses for the months of February, May, June, and August for southeastern US dairy cows. The months of May and June were selected because they represent a period of the year when grazing might be used and the weather transitions to summer. The months of February and August were chosen because they were representative months of winter and summer, respectively. In general, CR was greatest across all DIM in February, smallest in August, and intermediate in May and June.

An improvement in CR was observed from 25 to 50 DIM to 75 to 100 DIM for all months across all 3 birth-year groups. There was also a general increased improvement for CR across all 3 birth-year groups in May and June between 100 and 175 d; however, the increase in February was much smaller and nearly no change or even a decrease was observed in August.

In February and August, cows born recently and cows born in the past were very close in performance from 50 to 75 DIM to 150 to 175 DIM, with a range of difference between 0.3 and $4.5 \%$ across birth-year groups within month by DIM subclass. However, for the months of May and June, the range of differences in CR from 50 to 75 DIM to 150 to 175 DIM across birth-year groups within month by DIM subclass was much greater (1.4 to $15.1 \%$ ). Overall, it appeared that the deterioration over time in $\mathrm{CR}$ was much less in the 

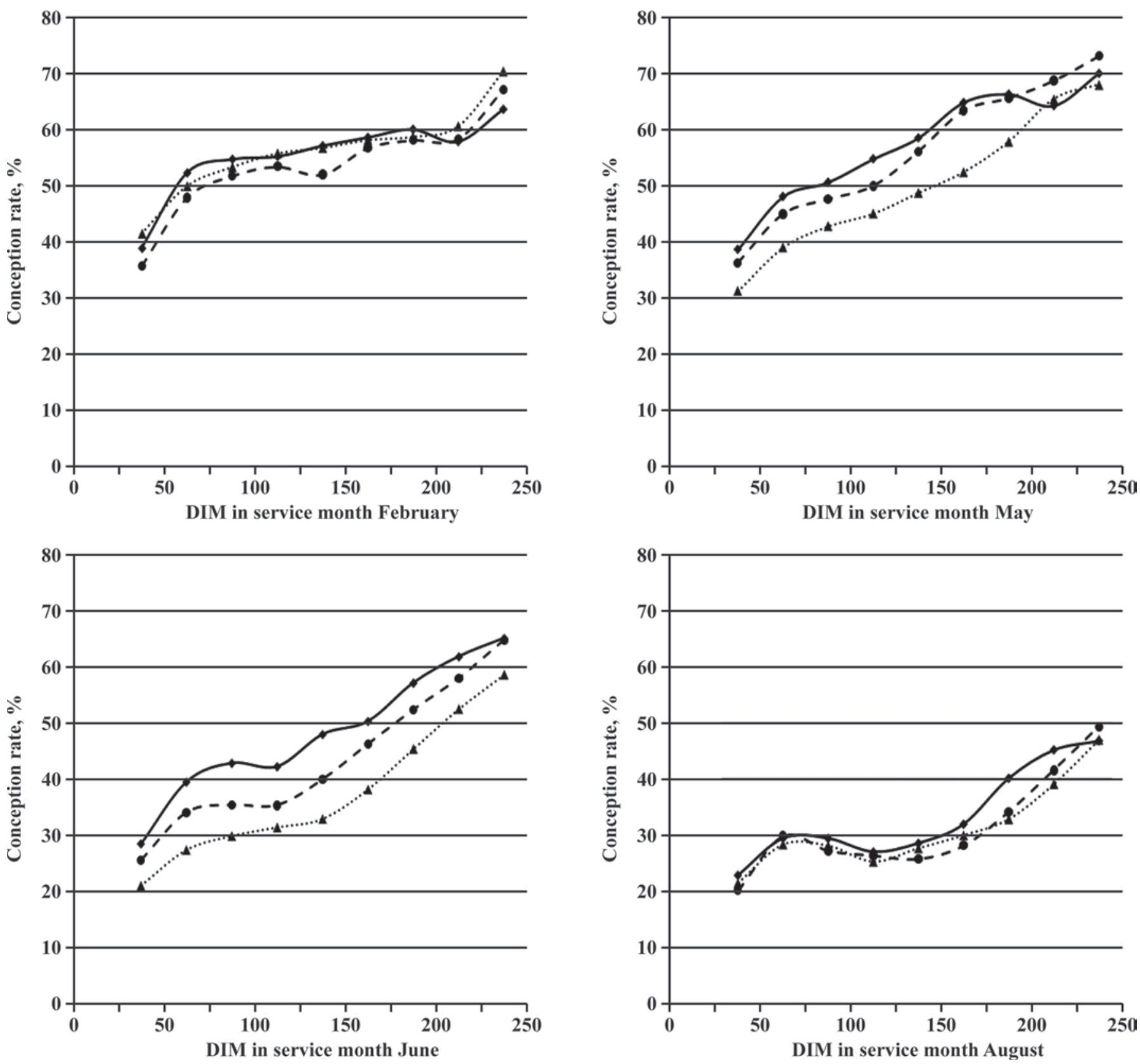

Figure 4. Conception rates for service month by DIM subclasses for the months of February, May, June, and August in the southeastern United States.

months of February and August compared with that in May and June. In May, the cows in recent periods returned to the same level of performance as cows with DIM of 75 to 100 or fewer days in the earlier period when the waiting period was 150 to $175 \mathrm{~d}$ and more. In June, these cows reached the fertility level of cows born earlier with DIM 50 to $75 \mathrm{~d}$ not before DIM of 175 to 200 d. That means that the CR of recent cows can be improved by appropriately increasing DIM.
Results obtained here for large DIM need to be viewed with caution, especially those $>175 \mathrm{~d}$, because they are likely to be biased because of the editing criteria. Higher CR at larger DIM may be justified by better energy balance and by improvements in the infection status of the uterus.

The CR trends presented in Figures 3 and 4 suggest that cows in the southeastern United States have declined in fertility over time in some seasons, and this 
reduction does not appear to be due solely to increases in milk production. Rather, it may indicate that cows have decreased in their ability to handle the switch to more pasture-based feeding systems or the onset of heat stress. Additional management protocols or genetic selection to combat the negative effects of heat stress may be necessary to halt, slow, or reverse the decline of cow reproduction in the future. Oseni et al. (2004) analyzed the information from 3 southeastern states and found that seasonal fluctuations in the cow fertility trait days open had a genetic component; therefore, selection of sires with daughters with lower days open within different seasons may be possible. High quality fertility data need to be collected and research needs to be conducted to explore the feasibility of a genetic evaluation for fertility that accounts for the effect of season and heat stress to identify bulls that can sire daughters with increased ability to reproduce under hot, humid conditions.

\section{CONCLUSIONS}

Conception rates for Holstein cows in the southeastern United States have appeared to decline over time during certain months (May and June). This decrease does not appear to be solely caused by increases in milk yield, but could signify that current cows have a reduced ability to handle the onset of heat stress and the switch to pasture-based management systems. Conception rate increased with increasing DIM in all birth-year groups; however, for some months (May and June), the increase did not return the CR levels of current cows to those of cows born in earlier years. Results of this observational study suggest that new management protocols in addition to the use of a VWP to breed and genetic selection to combat the negative effects of heat stress may be necessary to cope with the decline of cow fertility.

\section{ACKNOWLEDGMENTS}

Helpful comments by the 2 anonymous reviewers are gratefully acknowledged.

\section{REFERENCES}

Bohmanova, J., I. Misztal, and J. B. Cole. 2007. Temperature-humidity indices as indicators of milk production losses due to heat stress. J. Dairy Sci. 90:1947-1956.

Bohmanova, J., I. Misztal, S. Tsuruta, H. D. Norman, and T. J. Lawlor. 2008. Short communication: Genotype by environment interaction due to heat stress. J. Dairy Sci. 91:840-846.

Brouk, M. J., J. P. Harner, J. F. Smith, and D. V. Armstrong. 2007. Environmental modifications to address heat stress. J. Dairy Sci. 90(Suppl. 1):624. (Abstr.)

de Vries, A., and C. A. Risco. 2005. Trends and seasonality of reproductive performance in Florida and Georgia dairy herds from 1976 to 2002. J. Dairy Sci. 88:3155-3165.

Dematawewa, C. M. B., and P. J. Berger. 1998. Genetic and phenotypic parameters for 305-day yield, fertility, and survival in Holsteins. J. Dairy Sci. 81:2700-2709.

Huang, C., S. Tsuruta, J. K. Bertrand, I. Misztal, T. J. Lawlor, and J. S. Clay. 2008. Environmental effects on conception rate of Holsteins in New York and Georgia. J. Dairy Sci. 91:818-825.

López-Gatius, F. 2003. Is fertility declining in dairy cattle? A retrospective study in northeastern Spain. Theriogenology 60:8999.

Lucy, M. C. 2001. Reproductive loss in high-producing dairy cattle: Where will it end? J. Dairy Sci. 84:1277-1293.

Morton, J. M., W. P. Tranter, D. G. Mayer, and N. N. Jonsson. 2007. Effects of environmental heat on conception rates in lactating dairy cows: Critical periods of exposure. J. Dairy Sci. 90:2271-2278.

Oseni, S., I. Misztal, S. Tsuruta, and R. Rekaya. 2004. Genetic components of days open under heat stress. J. Dairy Sci. 87:30223028.

Pryce, J. E., and B. L. Harris. 2004. Genetic and economic evaluation of dairy cow body condition score in New Zealand. Interbull Bull. 32:82-85.

SAS Institute. 1996. SAS/STAT Software: Changes and Enhancements through Release 6.11. SAS Inst. Inc., Cary, NC.

Silvia, W. 1998. Changes in reproductive performance of Holstein dairy cows in Kentucky from 1972 to 1996. J. Dairy Sci. 81(Suppl. 1):244. (Abstr.)

Washburn, S. P., W. J. Silvia, C. H. Brown, B. T. McDaniel, and A. J. McAllister. 2002. trends in reproductive performance in southeastern Holstein and Jersey DHI herds. J. Dairy Sci. $85: 244-251$. 